\title{
THE UPS LOCUS \\ ENCODING UROPORPHYRINOGEN I SYNTHASE \\ IS LOCATED ON HUMAN CHROMOSOME 11
}

\begin{abstract}
M. Meisler ${ }^{1}$, L. Wanner ${ }^{1}$, R.E. Eddy ${ }^{2}$ and T.B. Shows ${ }^{2}$
${ }^{1}$ Department of Human Genetics, University of Michigan Medical School, Ann Arbor, Michigar 48109 and 'Biochemical Genetics Section, Roswell Park Memorial Institute, Buffalo, New York 14263
\end{abstract}

Received May 20,1980

Summary: The expression of the UPS locus encoding uroporphyrinogen I synthase has been investigated in human/mouse somatic cell hybrids. Human and mouse uroporphyrinogen I synthase can be readily distinguished by their isoelectric points. In hybrid cells, both human and mouse isozymes are detected. The multiple human uroporphyrinogen I synthasc isozymes segregate as a single unit, as expected if they are the products of a single locus. The absence of new heteropolymers in hybrid cells supports the biochemical evidence that the active enzyme is a monomer. The presence of human uroporphyrinogen I synthase in hybrid clones was correlated with the presence of human chromosome 11, or its enzymatic marker, without exception in 44 independent hybrid lines. All other chromosomes could be eliminated as possible locations for this locus, due to their independent segregation. This report represents the first gene assignment for an enzyme in the heme biosynthesis pathway.

Uroporphyrinogen I synthase (EC 4.3.1.8) is the third enzyme in the pathway leading to the biosynthesis of heme. Partial deficiency of this enzyme is associated with the human disorder Acute Intermittent Porphyria, an autosomal dominant inherited disease (1). Deficiencies of the other enzymes of heme biosynthesis also produce dominantly-inherited disorders (1). Chromosomal linkage data for these enzymes would make possible improved diagnosis of these disordcrs through the analysis of closely-linked genetic markers. The linkage relationships among these functionally related enzymes would also be of interest.

We have recently described a simple isoelectric focusing method for the analysis of uroporphyrinogen I synthase isozymes in tissue extracts (2). Human and mousc tissues contain multiple isozymes, and additional erythrocyte specific isozymes are present in both species. These isozymes have recently This work was supported by Contract $E$ (11-1) (2828) from the U.S. Department of Energy and by USPHS Grants GM 24872, GM 20454 and HD 05196. 
been observed in a highly purified enzyme preparation from human erythrocytes

(3). Anderson and Desnick present evidence that the isozymes are stable

intermediates in the stepwise condensation of four molecules of porphobilinogen (3).

Genetic transmission of a structural variant of uroporphyrinogen I synthase through three generations demonstrated the autosomal linkage of the human UPS locus (2). Genetic analysis in mice revealed that all of the mouse isozymes are encoded by a single autosomal locus, designated Ups (2). In the present study, we have used the difference in iscelectric points of the human and mouse enzymes to study their expression in interspecific cell hybrids. Analysis of isozyme patterns in these cells has provided new information regarding the genetic linkage and biochemical properties of this enzyme.

METHODS:

Cell Lines: Human-mouse somatic cell hybrids were isolated from the fusion of human fibroblasts with RAG (HPRT'), IM/TK or A9 (HPRT') mouse cells as described (4). Cell fusions were carried out with either inactivated Sendai virus (4) or polyethylene glycol (5). Cell hybrid clones were isolated and maintained in Dulbecco's modified Eagle's medium (GIBCO) containing hypoxanthine, aminopterin, thymidine, 10 z fetal calf serum, and antibiotics (4). Human parental fibroblasts of normal karyotype were WI-38 (ATCC \#CCL75), GM654, GM469, and SH421. Human parental fibroblasts carrying reciprocal translocations for regional mapping were AnLy (carrying an $x / 9$ translocation), JoSt (carrying a $7 / 9$ translocation), DUV (carrying an $x / 15$ translocation), GM2808 (carrying a $3 / 17$ translocation), and GM194 (carrying an $\mathrm{X} / 3$ translocation). Fibroblasts with a GM prefix were obtained from Human Genetic Mutant Cell Repository, Camden, N.J. The cell hybrids utilized were from the independent hybrid sets: WIL (WI-38 $\mathrm{LLM}^{-T^{-}}{ }^{-}$), ALR (AnLY $\mathrm{x}$ RAG), JSR (JoSt $\mathrm{x}$ RAG), DUA (DUV $x$ A9), TSL (GM2808 $x$ LM/TK'), MAR (GM654 x RAG), SIR (GM469 $x$ RAG), RAS (SH421 x RAG), and XTR (GM194 $\times$ RAG). Additional information regarding these hybrid sets has been published (6). Cell hybrids were harvested and homogenized as described (4) at $10^{8}$ cells/ml in $0.05 \mathrm{M}$ Tris ( $\mathrm{pH}$ 7.5). The hybrids analyzed were derived from nine diffcrent individuals and three independent rodent cell lines. The $Y$ chromosome was excluded from consideration since autosomal Iinkage of the UPS locus has been demonstrated in family studies (2); furthermore the isozyme pattern of human parental cell lines from females are normal. The human liver extract was prepared as described previously (2).

Isoelectric Focusing of Uroporphyrinogen $T$ Synthase: Sixty to ninety microliter aliquots of soluble extracts from cultured cells or tissues were applied with filter paper applicators to the surface of LKB PAG-Plates, $\mathrm{pH} 4.0$ - 6.5. Ten samples were applied across the narrow dimension near the basic end of each gel; focusing was carried out for $21 / 2$ hours at the maximum voltage recommended by the manufacturer. After focusing, gels were stained for activity (2) by incubation for $45-90 \mathrm{~min}$ at $45^{\circ}$ in the presence 
of $90 \mu \mathrm{M}$ porphobilinogen (Sigma) in $0.3 \mathrm{M} \mathrm{Tris-HCl,} \mathrm{pH} 8.2$. The red fluorescent bands, resulting from the enzymatic production of uroporphyrinogen and its spontaneous oxidation to uroporphyrin, were photographed with ultraviolet illumination. Values of isoelectric points were determined as described (2) and varied by up to $0.1 \mathrm{pH}$ unit in separate experiments.

\section{RESULTS:}

The uroporphyrinogen I synthase isozyme patterns of mouse and human cells are quite different. Mouse cells contain three major regions of activity which focus between pH 5.3 and pH 5.1 (Figure 1, Ianes 2 and 8 ). The human isozymes vary in isoelectric point from $\mathrm{pH} 5.3$ to $\mathrm{pH} 5.8$, with the major activity band at pH 5.8 (Eigure 1 , lanes 1 and 9). When cloned hybrid cells were examined, the existence of two classes of hybrids was evident. Clones classified "negative" for human UPS contained isozyme patterns

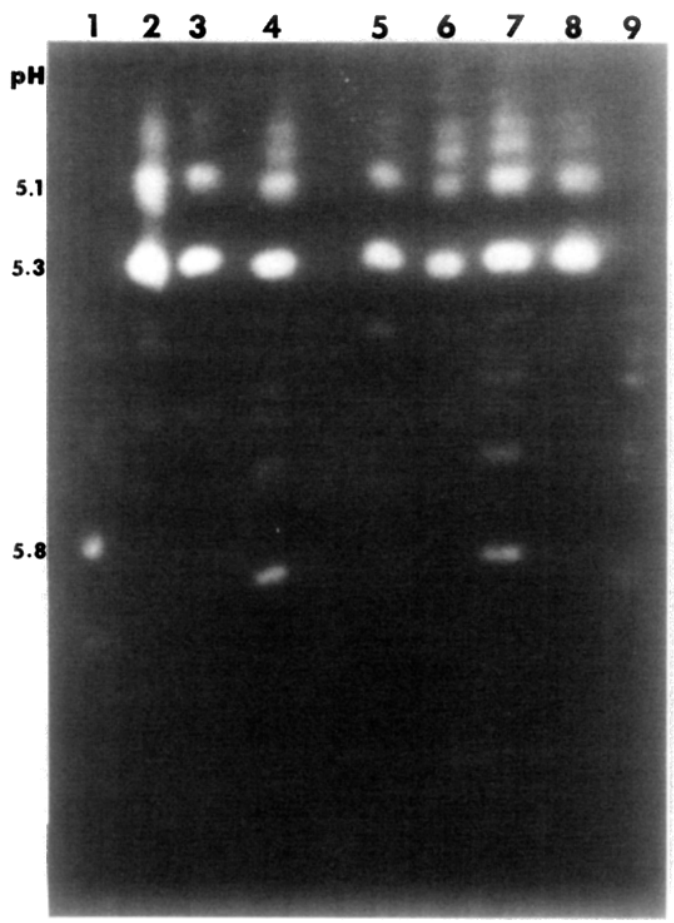

Fig 1. Isozyme Patterns of Human, Mouse, and Interspecific Hybrid Cells. Samples were focused on $24 \mathrm{~cm}$ PAG plates, pH $4.0-6.5$. Uroporphyrinogen I synthase activity was stained as described in the text. Lane 1 , human liver extract; Lane 2, a mixture of mouse lines RAG and LM/TK, 1:1; Lane 3, negative hybrid line wil-1; Lane 4, human UPS positive hybrid line Wil-8x; Lane 5, mouse line IM/TK ; Lane 6, human UPS negative hybrid DUA-8; Lane 7, human UPS positive hybrid line wil-8x; Lane 8 , mouse fibroblast line RAG; Lane 9, human 1ymphoblast cell line uM56. 
Table I. Segregation of Human Chromosome Markers and Human Uroporphrinogen I Synthase in Somatic Cell Hybrids. The presence of human enzyme markers of known chromosomal linkage was determined by electrophoretic analysis (7-9). Concordant segregation columns indicate the number of ciones in which human UPS isozymes and all enzymes in a linkage group were either present or absent together. Discordant segregation columns show the number of clones in which only the enzyme markers or UPS were present.

\begin{tabular}{|c|c|c|c|}
\hline Chromosome & Enzyme Markers & Concordant & Discordant \\
\hline 1 & AK2, PEPC, PGM1 & 22 & 10 \\
\hline 2 & IDHl, MDHl & 16 & 16 \\
\hline 3 & ACY 1 & 25 & 8 \\
\hline 4 & PEPS & 19 & 9 \\
\hline 5 & $\mathrm{HEXB}$ & 23 & 9 \\
\hline 6 & $\mathrm{ME} 1, \mathrm{SOD} 2$ & 20 & 12 \\
\hline 7 & GUSB & 23 & 9 \\
\hline 8 & GSR & 17 & 14 \\
\hline 9 & $\mathrm{AKl}$ & 19 & 14 \\
\hline 10 & GOTl & 24 & 8 \\
\hline 11 & LDHA & 33 & 0 \\
\hline 12 & $\mathrm{IDHB}, \mathrm{PEPB}$ & 18 & 14 \\
\hline 13 & ESD & 20 & 11 \\
\hline 14 & $\mathrm{NP}$ & 23 & 9 \\
\hline 15 & MPI, PKM2 & 12 & 20 \\
\hline 16 & APRT & 22 & 9 \\
\hline 17 & GALK & 16 & 11 \\
\hline 18 & PEPA & 24 & 8 \\
\hline 19 & GPI & 19 & 12 \\
\hline 20 & $\mathrm{ADA}$ & 21 & 11 \\
\hline 21 & SODl & 23 & 9 \\
\hline 22 & $\mathrm{ACO} 2$ & 12 & 9 \\
\hline$x$ & G6PD, PGK & 24 & 9 \\
\hline
\end{tabular}

indistinquishable from the mouse parental lines (Figure 1, lanes 3 and 6). The positive hybrid clones contained, in addition to the mouse isozymes, the complete set of human isozymes including the intensely staining band at pH 5.8 (Figure I, lanes 4 and 7). Because the pH gradient is very shallow, we obtain a separation distance of $5 \mathrm{~cm}$ between the major human and mouse isozymes, corresponding to a difference in isoelectric point of $0.5 \mathrm{pH}$ units. All hybrids classified as positive for human UPS contained both the intense isozyme at $\mathrm{pH} 5.0$ and the minor isozymes between $\mathrm{pH} 5.0$ and $\mathrm{pH} 5.3$. In hybrids classified as negative, there was no visible activity in the gel 
region more basic than pH 5.3. A few hybrids with faint activity more basic than $\mathrm{pH} 5.3$ were excluded from the analysis.

Thirty-three hybrid cell lines were examined for the presence or absence of human uroporphyrinogen I synthase as well as 30 other human enzymes (Table I). These enzyme markers represent linkage groups which have been assigned to the 23 human autosomes and to the $x$ chromosome (8). Human uroporphyrinogen I synthase isozymes segregated concordantly with the lactate dehydrogenase-A (IDHA) marker (4) encoded on chromosome 11 (10) and independently of all other enzyme markers. These data demonstrate the linkage of the UPS and IDHA loci. The assignment of the UPS locus to chromosome 11 was supported by the analysis of 11 additional clones for which karyotypes were available (Table II). In these hybrids, human UPS was expressed only when chromosome 11 was retained. All other chromosomes showed discordant segregation with UPS, excluding them from consideration. Thus, both enzyme marker and chronosome analyses support the assignment of UPS to human chromosome 11 .

\section{DISCUSSION:}

We have observed the cosegregation of human UPS isozymes with chromosome 11 and a chromosome 11 enzyme marker in human-mouse hybrid cell lines. The concordancy rate of $11 / 11$ with the chromosome in karyotyped lines, and $33 / 33$ with the LDH A marker in additional hybrid lines, justify the assignment of the UPS locus to human chromosome 11 .

The isozyme patterns of the interspecific hybrid cells provide two additional types of information. First, we did not observe the formation of extra heteropolymers of intermediate isoelectric point; rather, the isozyme pattern of positive hybrids appeared to be simply additive of the two parental lines. The apparent absence of hybrid heteropolymers molecules supports the biochemical evidence that the active enzyme is a monomer, since in cases of multi-subunit enzymes, interspecific hybrid isozymes of intermeaiate charge are observed. Second, we observed co-segregation of the entire set of human 


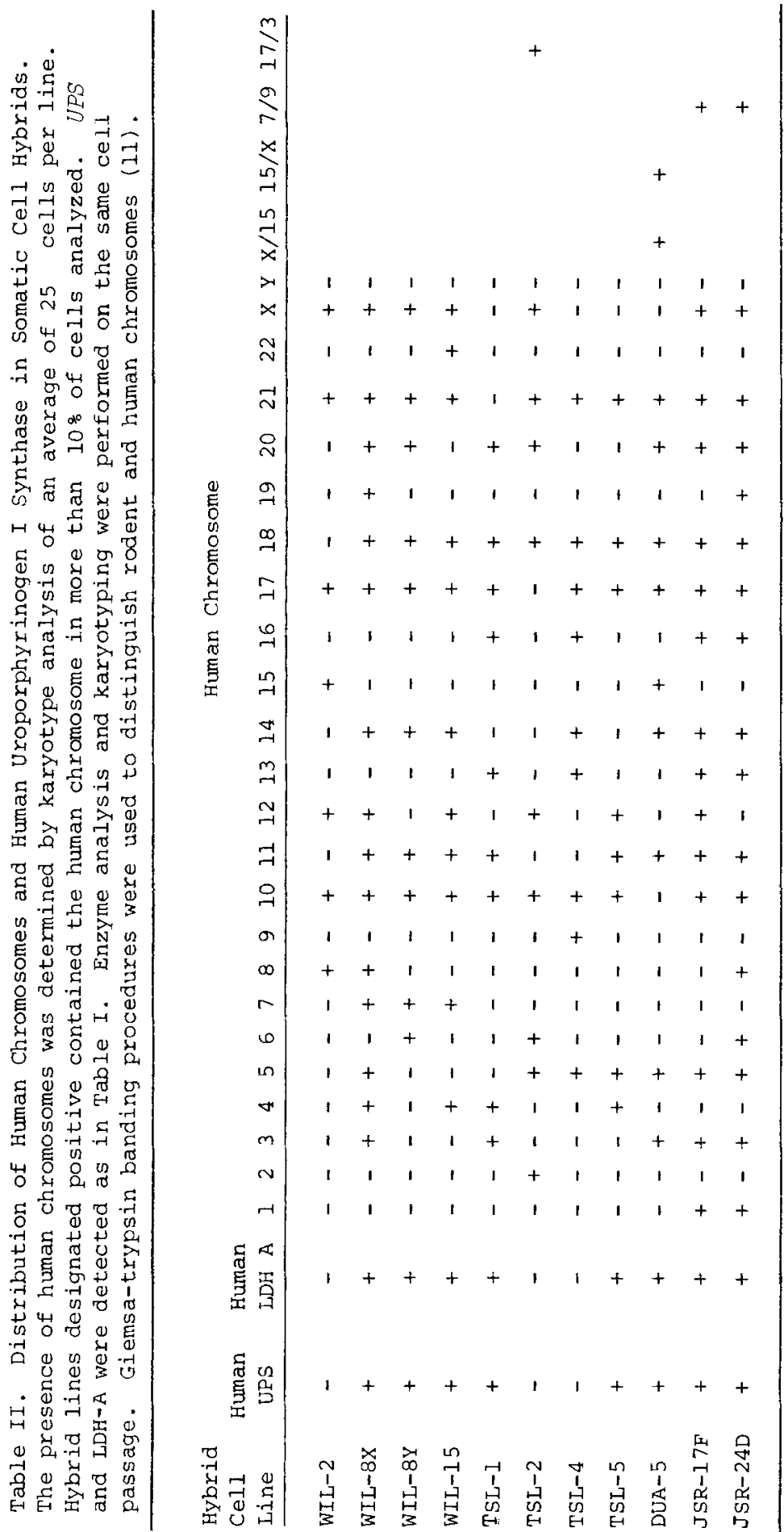


isozymes as a genetic unit, demonstrating that they are the products of a single genetic locus.

It will be of great interest to map the genes encoding the other enzymes of the heme biosynthesis pathway. It is relevant to considerations of molecular evolution of this pathway to note that the conversion of porphobilinogen to uroporphyrin can proceed spontaneously under moderate conditions (12). If the spontaneous process in primitive cells preceeded the recruitment of enzymes to catalyze the reactions, we might not anticipate any Iinkage among the subsequent enzymes of the pathway. In $\underline{E}$. coli, uroporphyrinogen I synthase and cosynthase are closely linked (13), while the other genes are unlinked. It is also of interest that the gene encoding the $\beta$ chain of human hemoglobin is located on Chromosome 11.

Acknowledgement: The exceltent assistance of L. Halzey, $M$. Byers and C. Young is gratefulty acknowledged. The contributions and advice of $D r$. J. Brown and D. Shaver on the original kayyotypic analyses of the cell hybrids is very mich appreciated.

\section{References :}

1. Meyer, U.S., and Schmid, R. (1978) in The Metabolic Basis of Inherited Disease, pp. 1166-1221, eds. Stanbury, J.B., Wyngaarden, J.B. and Fredrickson, D.S. (MCGraw Hill), 4th edition.

2. Meisler, M., and Carter, M. (1980) Proc. Nat1. Acad. Sci. 77, in press.

3. Anderson, P.M., and Desnick, R.J. (1980) J. Biol. Chem. 255, 1993-1999.

4. Shows, T.B. (1972) Proc. Natl. Acad. Sci. 69, 348-352.

5. Davidson, R.L., and Gerald, P.S. (1976) Somatic Cell Genet. 2, $165-176$.

6. Koch, G.A., and Shows, T.B. (1980) proc. Natl. Acad. Sci. 77, in press.

7. Shows, T.B. (1977) in Isozymes: Current Topics in Biological and Medical Research, pp. 107-158, Vol. 2, Eds. M.C. Rattazzi, J.G. Scandalios, and G.S. Whitt. Alan R. Liss, New York.

8. Shows, T.B., and MCAlpine, P.J. (1979) Cytogenet. Cell Genet. 25, 117127.

9. Naylor, S.L., Klebe, R.J., and Shows, T.B. (1978) Proc. Natl. Acad. Sc1. $75,6159-6162$.

10. Shows, T.B., Brown, J.A., and Lalley, P.A. (1976) Cytogenet. Cell Genet. $16,231-234$.

11. Shows, T.B., and Brown, J.A. (1975) Proc. Natl. Acad. Sci. 72, 2125-2129.

12. Hendry, G.A.F., and Jones, O.T.G. (1980) J. Med. Genet. 17, 1-14.

13. Bachmann, B.J., and Low, K.B. (1980), Microbiol. Rev. 44, 1-56. 